\title{
The Principle of Strong Diminishing Transfer*
}

\author{
Alain Chateauneuf $\ddagger^{\dagger}$ Thibault Gajdos $\stackrel{\ddagger}{\ddagger}$ Pierre-Henry Wilthien ${ }^{\S}$
}

September 2000

\begin{abstract}
We reconsider the principles of diminishing transfer (introduced by Kolm (1976)) and dual diminishing transfer (introduced by Mehran (1976)). It appears that if a Rank Dependent Expected Utility (RDEU) maximizer respects the principle of diminishing (resp. dual diminishing) transfer, then he behaves in accordance with the Expected Utility model (resp. Yaari's dual model).

This leads us to define the principle of strong diminishing transfer, which is a combination of the principles of diminishing and dual diminishing transfer. We give necessary conditions for a RDEU maximizer to respect this principle.

These results are applied to the problem of inequality measurement. Journal of Economic Literature Classification Number: D63

Key words : Inequality Measurement, Principle of Diminishing Transfers.
\end{abstract}

\section{Introduction}

The well-known Pigou-Dalton transfer principle requires that any transfer of income from a richer to a poorer individual, which does not reverse which of the two is richer, reduces inequality. In a seminal paper, Kolm (1976) goes a step further, and introduces the principle of diminishing transfer. This principle requires that "one values more such a transfer between persons with given income difference if these incomes are lower than if they are higher" (Kolm, (1976), p.87).

It is well-known that the Atkinson, Kolm and Theil indices respect this principle. It is obviously not the case for the Gini index, since its sensitivity depends not on the income levels of the individuals concerned by the transfer, but on their ranks. This has been seen as a limit of the Gini index ${ }^{1}$. But, on the other hand, one may argue that the Gini index respects another principle, which is not satisfied by the Atkinson, Kolm and Theil indices: The Gini

\footnotetext{
${ }^{*}$ We thank an anonymous referee of the Journal of Economic Theory for very helpful comments and suggestions.

†Corresponding author. CERMSEM, Université Paris 1 Panthéon Sorbonne, 106-112 Boulevard de L'Hôpital 75647 Paris cedex 13. E-mail : chateaun@univ-paris1.fr

${ }^{\ddagger}$ EUREQua Université Paris 1 Panthéon-Sorbonne and ESSEC, 106-112 boulevard de L’Hôpital 75647 Paris cedex 13. E-mail: gajdos@univ-paris1.fr

${ }^{\S}$ CERMSEM, Université Paris 1 Panthéon Sorbonne, 106-112 Boulevard de L'Hôpital 75647 Paris cedex 13. E-mail : ph@wilthien.com

${ }^{1}$ See, e.g., Sen (1973).
} 
index is more sensitive for Pigou-Dalton transfers between persons with given rank difference if these ranks are lower than if they are higher. Let us call this principle, introduced by Mehran (1976) and Kakwani (1980), the principle of dual diminishing transfer. At first sight, there is no obvious reason to prefer one principle to the other, and one may even want both to be satisfied. Admittedly, this is a matter of taste. Nevertheless, and surprisingly enough, whereas a number of papers are devoted to the principle of diminishing transfer (see, among others, Kolm (1976), Shorrocks and Foster (1987), Davies and Hoy (1994)), little have been written about the principle of dual diminishing transfer ${ }^{2}$.

Obviously, the principle of dual diminishing transfer is only relevant if one cares about individuals' ranks. Therefore, these concepts have no meaning in the von Neumann-Morgenstern model. On the contrary, they are relevant in Yaari's dual model (1987) and its generalization, Quiggin's Rank Dependent Expected Utility Model (1982).

The aim of this paper is hence to characterize the principles of diminishing transfer and of dual diminishing transfer in Yaari's and Quiggin's models. We give necessary and sufficient conditions for a decision maker who behaves in accordance with Yaari's dual model to respect the principle of dual diminishing transfer. Unfortunately, it appears that if a decision maker who behaves in accordance with the Rank Dependent Expected Utility model respects the principle of diminishing transfer, then he behaves in accordance with the Expected Utility model. Similarly, if such a decision maker respects the principle of dual diminishing transfer, then he behaves in accordance with Yaari's dual model.

This leads us to define the principle of strong diminishing transfer, which states that a transfer from an individual with rank $i$ and income $x$ to one with rank $i-p$ and income $x-\Delta$ has a greater impact the lower $i$ and $x$ are. Whereas it is unclear if one should prefer the principle of diminishing transfer or the principle of dual diminishing transfer, it seems that, at least, one should agree with the principle of strong diminishing transfer (obviously, if the decision maker respects the principle of diminishing transfer or the principle of dual diminishing transfer, he also respects the principle of strong diminishing transfer - the converse, however, is not true). Hence, it seems that the principle of strong diminishing transfer does really make sense. Furthermore, we give necessary and sufficient conditions for a decision maker who behaves in accordance with the Rank Dependent Expected Utility model to respect the principle of transfer and the principle of strong diminishing transfer. It should be emphazised that, although the various transfer principles defined in this paper only apply to comparisons of distributions with the same population size, the results we establish are variable-population theorems. Actually,

\footnotetext{
${ }^{2}$ See however the related notion of "favourable double transfer" in Chateauneuf and Wilthien (1999), and Zoli's independant paper (1999).
} 
it is important for some of the proofs that the set of income distributions is not restricted to a fixed population size. Finally, these results are applied to the measurement of inequality.

The organization of this paper is as follows. In section 2 we introduce notation and some definitions. Section 3 contains our main results, which are applied to the problem of inequality measurement in section 4.

\section{Notation and definitions}

Let $\Omega$ be the set of rank-ordered uniform and discrete income distributions, with values in $\mathbb{R}_{+}$. An income distribution $X \in \Omega$ is defined by:

$$
X=\left(x_{1}, \frac{1}{n} ; x_{2}, \frac{1}{n} ; \ldots ; x_{n}, \frac{1}{n}\right)
$$

with $0 \leq x_{1} \leq x_{2} \leq \ldots \leq x_{n}$. Note that for any income distribution $Y=\left(y_{1}, p_{1} ; y_{2}, p_{2} ; \ldots ; y_{k}, p_{k}\right)$ where the $p_{i}$ are rational numbers and $\sum_{i=1}^{k} p_{i}=1$ there exists $m \geq 2$ such that $Y=\left(y_{1}, \frac{1}{m} ; y_{2}, \frac{1}{m} ; \ldots ; y_{m}, \frac{1}{m}\right)$.

For simplicity, we let $X=\left(x_{1}, x_{2}, \ldots, x_{n}\right) . \bar{X}$ denotes the mean of the income distribution $X$, and $e_{i}$ denotes the $n$-tuple $(0, \ldots, 0,1,0, \ldots, 0)$ whose only non-zero element occurs in the $i$-th position.

\section{$2.1 \quad$ Preferences}

Let $\succeq$ be the decision maker's preference relation over $\Omega$. We call here a "decision maker" anybody behind the veil of ignorance. This assumption is standard in the field of normative inequality measurement. A decision maker behaves in accordance with the Expected Utility model (EU model) if there exists a strictly increasing utility function $u: \mathbb{R}_{+} \rightarrow \mathbb{R}$ such that $\succeq$ is represented by:

$$
U(X)=\sum_{i=1}^{n} \frac{1}{n} u\left(x_{i}\right) .
$$

A decision maker behaves in accordance with Yaari's dual model if there exists a strictly increasing continuous probability transformation $f:[0,1] \rightarrow[0,1]$ with $f(0)=0$ and $f(1)=1$ such that $\succeq$ is represented by:

$$
W(X)=\sum_{i=1}^{n}\left[f\left(\frac{n-i+1}{n}\right)-f\left(\frac{n-i}{n}\right)\right] x_{i} .
$$

Finally, a decision maker behaves in accordance with Quiggin's Rank Dependent Expected Utility Model (RDEU model) if there exists a strictly increasing utility function $u: \mathbb{R}_{+} \rightarrow \mathbb{R}$ and a strictly increasing continuous probability transformation $f:[0,1] \rightarrow[0,1]$ with $f(0)=0$ 
and $f(1)=1$ such that $\succeq$ is represented by:

$$
W(u(X))=\sum_{i=1}^{n}\left[f\left(\frac{n-i+1}{n}\right)-f\left(\frac{n-i}{n}\right)\right] u\left(x_{i}\right) .
$$

In the sequel, we let for any $i \in\{1, \ldots, n\}: \Psi\left(\frac{i}{n}\right)=f\left(\frac{n-i+1}{n}\right)-f\left(\frac{n-i}{n}\right)$.

Following Kolm (1969), Atkinson (1970) and Sen (1973), one can derive an inequality measure from a social welfare function. Let $\Xi(X)$ be the per capita income which, if distributed equally, is indifferent to $X$ according to the social welfare function $W$. This "equally distributed equivalent income" is implicitly defined by the relation:

$$
W(X)=W(\Xi(X) e)
$$

where $e$ denotes the unit vector of $\mathbb{R}^{n}$. It is then possible to define a relative inequality index:

$$
I(X)=1-\frac{\Xi(X)}{\bar{X}}
$$

and an absolute inequality index:

$$
J(X)=\bar{X}-\Xi(X) .
$$

The Gini index — which may be either absolute or relative — and its generalizations are based on a rank dependent social welfare function.

\subsection{The principle of strong diminishing transfers}

Let us now define the well known principle of transfer, the principle of diminishing transfer and the principle of dual diminishing transfer.

Definition 1 A decision maker respects the principle of transfer if for all $X$ belonging to $\Omega, i_{1}<$ $i_{2}$, and $\varepsilon>0$ such that $x_{i_{1}}+\varepsilon \leq x_{i_{1}+1}$ and $x_{i_{2}-1} \leq x_{i_{2}}-\varepsilon$ :

$$
X_{1}=X+\left(e_{i_{1}}-e_{i_{2}}\right) \varepsilon \succeq X
$$

Definition $2 A$ decision maker respects the principle of diminishing transfer if for all $X$ belonging to $\Omega, i_{1}<i_{2}, i_{3}<i_{4}, i_{1}<i_{3}$ and all $\varepsilon>0$ such that $x_{i_{1}}+\varepsilon \leq x_{i_{1}+1}, x_{i_{2}-1} \leq x_{i_{2}}-\varepsilon$, $x_{i_{3}}+\varepsilon \leq x_{i_{3}+1}, x_{i_{4}-1} \leq x_{i_{4}}-\varepsilon, x_{i_{2}}-x_{i_{1}}=x_{i_{4}}-x_{i_{3}}$,

$$
X_{1}=X+\varepsilon\left(e_{i_{1}}-e_{i_{2}}\right) \succeq X+\varepsilon\left(e_{i_{3}}-e_{i_{4}}\right)=X_{2}
$$


Hence a decision maker respects the principle of diminishing transfer if a transfer from an individual with income $x$ to one with income $x-\Delta$ (with $\Delta>0$ ) has a greater impact on social welfare the lower $x$ is.

This principle is illustrated on Figure 1. We start from the distribution represented by a continuous line. We then construct two distributions: One is obtained using a Pigou-Dalton transfer from the second poorest individual to the poorest one (thin dotted line), and the other is obtained using a Pigou-Dalton transfer of the same amount $\varepsilon$ from the richest individual to the third richest individual (thick dashed line). Here, $x_{5}-x_{3}=x_{2}-x_{1}$. Hence, if one agrees with the principle of diminishing transfer, the first distribution is preferred to the second one.

Include figure 1 : The principle of diminishing transfer

The principle of diminishing transfer raises a problem, which is illustrated by Figure 2 .

Include figure 2 : First objection to the principle of diminishing transfer

Here, we start from the income distribution represented by a thick line. Now, we construct two distributions: One is obtained using a Pigou-Dalton transfer of amount $\varepsilon$ from the second poorest individual to the poorest one (thin dashed line), and the other is obtained using to a Pigou-Dalton transfer of amount $\varepsilon$ from the richest individual to the second poorest one (thick dashed line). Since $x_{8}-x_{2}=x_{2}-x_{1}$, if one agrees with the diminishing transfer principle, one should prefer the first transfer to the second one. But, in the first case, we take money from the second poorest individual, whereas in the second case we take money from the richest one. Moreover, in the first case, the share of the two poorest individuals' income in the total income remains constant, whereas it increases in the second case.

Assume, now, that we evaluate income distributions through an additively separable evaluation function which respects the principle of diminishing transfer (e.g., a von NeumannMorgenstern functional with a utility function $u$ such that $u^{\prime}(x)>0$ and $\left.u^{\prime \prime \prime}(x)>0\right)$. Then a new problem arises, as shown by Figure 3 .

Include figure 3 : second objection to the principle of diminishing transfer

Here, there are two distributions, one represented by a thick line $(X)$ and the other represented by a thin line $(Y)$. In both distributions, we consider a Pigou-Dalton transfer of amount $\varepsilon$, from $x_{3}$ to $x_{1}$ and from $y_{7}$ to $y_{5}$, respectively. Since $x_{3}-x_{1}=y_{7}-y_{5}$ and $y_{5}<x_{1}$, the gain (evaluated by an evaluation function which respects the diminishing transfer principle) of the transfer in the distribution $Y$ is higher than the one of the transfer in the distribution $X$. But in 
the distribution $X$ the transfer concerns the poorest individual, whereas it concerns the fourth richest one in the distribution $Y$.

All this leads us to introduce the principle of dual diminishing transfer, which is defined as follows:

Definition $3 A$ decision maker respects the principle of dual diminishing transfer if for all $Y$ belonging to $\Omega, i_{1}<i_{3}, i_{1}-i_{2}=i_{3}-i_{4}<0$, and all $\varepsilon>0$ such that $y_{i_{1}}+\varepsilon \leq y_{i_{1}+1}, y_{i_{2}-1} \leq y_{i_{2}}-\varepsilon$, $y_{i_{3}}+\varepsilon \leq y_{i_{3}+1}, y_{i_{4}-1} \leq y_{i_{4}}-\varepsilon$

$$
Y_{1}=Y+\varepsilon\left(e_{i_{1}}-e_{i_{2}}\right) \succeq Y+\varepsilon\left(e_{i_{3}}-e_{i_{4}}\right)=Y_{2}
$$

Hence, a decision maker respects the principle of dual diminishing transfer if a transfer from an individual with rank $i$ to one with rank $i-p$ has a greater impact on social welfare the lower $i$ is. This principle is illustrated on the following Figure.

\section{Include Figure 4 : The principle of dual diminishing transfer}

Of course, the principle of dual diminishing transfer is also debatable, since it does not take into account the gap of income between individuals concerned by the transfer. Hence, there is no obvious reason to prefer one principle to the other. Admittedly, that is a matter of taste.

Therefore, we define the principle of strong diminishing transfer as follows:

Definition 4 A decision maker respects the principle of strong diminishing transfer if for all $Z$ belonging to $\Omega, i_{1}<i_{3}, i_{1}-i_{2}=i_{3}-i_{4}<0$ and all $\varepsilon>0$ such that $z_{i_{1}}+\varepsilon \leq z_{i_{1}+1}, z_{i_{2}-1} \leq z_{i_{2}}-\varepsilon$, $z_{i_{3}}+\varepsilon \leq z_{i_{3}+1}, z_{i_{4}-1} \leq z_{i_{4}}-\varepsilon, z_{i_{2}}-z_{i_{1}}=z_{i_{4}}-z_{i_{3}}$,

$$
Z_{1}=Z+\varepsilon\left(e_{i_{1}}-e_{i_{2}}\right) \succeq Z+\varepsilon\left(e_{i_{3}}-e_{i_{4}}\right)=Z_{2}
$$

Whereas it is unclear if one should prefer the principle of diminishing transfer or the principle of dual diminishing transfer, it seems that, at least, one should agree with the principle of strong diminishing transfer (obviously, if the decision maker respects the principle of diminishing transfer or the principle of dual diminishing transfer, he also respects the principle of strong diminishing transfer - the converse, however, is not true). Hence, it seems that the principle of strong diminishing transfer does really make sense.

By definition, $X_{1} \succeq X_{2}$ (resp. $Y_{1} \succeq Y_{2}$ or $\left.Z_{1} \succeq Z_{2}\right)$ is equivalent to $W\left(X_{1}\right) \geq W\left(X_{2}\right)$ (resp. $W\left(Y_{1}\right) \geq W\left(Y_{2}\right)$, or $W\left(Z_{1}\right) \geq W\left(Z_{2}\right)$ ) which, since $\bar{X}_{1}=\bar{X}_{2}, \bar{Y}_{1}=\bar{Y}_{2}$, and $\bar{Z}_{1}=\bar{Z}_{2}$ is equivalent to $I\left(X_{1}\right) \leq I\left(X_{2}\right)$ and $J\left(X_{1}\right) \leq J\left(X_{2}\right)$ (resp. $I\left(Y_{1}\right) \leq I\left(Y_{2}\right)$ and $J\left(Y_{1}\right) \leq J\left(Y_{2}\right)$, and $I\left(Z_{1}\right) \leq I\left(Z_{2}\right)$ and $\left.J\left(Z_{1}\right) \leq J\left(Z_{2}\right)\right)$. We will therefore, in the sequel, restrict our attention to the social welfare functions $W$. 
Note that all the present discussion may be extended to the problem of decision under risk, interpreting $W$ as an individual utility function and $\Omega$ as a set of lotteries. In this context, respecting the principle of diminishing transfer is similar to prudence (see, e.g., Kimball (1990), Eeckhoudt, Gollier and Schneider (1995)). Similarly respecting the principle of dual diminishing transfer may be translated in the field of risk aversion.

\section{Characterization of the principles of diminishing transfer and dual diminishing transfer}

In the sequel, we consider a utility function $u$, continuous on $\mathbb{R}_{+}$, strictly increasing on $\mathbb{R}_{+}$and three times differentiable on $\mathbb{R}_{++}$, and a frequency transformation function $f$, continuous on $[0,1]$, strictly increasing on $[0,1]$, three times differentiable on $] 0,1[$ and such that $f(0)=0$ and $f(1)=1$.

Our main results are as follows.

Theorem 1 For a decision maker who behaves in accordance with the Expected Utility model, the two following propositions are equivalent:

(i) the decision maker respects the principle of diminishing transfer,

(ii) $u^{\prime \prime \prime}(x) \geq 0$ for all $x$ in $\mathbb{R}_{++}$.

Proof: See the appendix.

This result is already known when $u$ is concave (see, e.g., Kolm (1976) and Eeckhoudt, Gollier and Schneider. (1995)). It appears that indeed no condition on the concavity of $u$ is needed to prove this result.

Theorem 2 For a decision maker who behaves in accordance with Yaari's dual model, the two following propositions are equivalent:

(i) the decision maker respects the principle of dual diminishing transfer,

(ii) $f^{\prime \prime \prime}(p) \geq 0$ for all $p$ in $] 0,1[$.

Proof: See the appendix.

Note that a similar result can be found in Mehran (1976) for continuous income distributions.

One may wonder under what conditions a decision maker who behaves in accordance with the RDEU model respects the principle of diminishing transfer or the principle of dual diminishing transfer. Unfortunately, as shown by the two following theorems, it appears that if such a decision 
maker respects the principle of diminishing transfer, then he behaves in accordance with the EU model. On the other hand, if he respects the principle of dual diminishing transfer, then he behaves in accordance with Yaari's dual model.

Theorem 3 If a decision maker who behaves in accordance with the RDEU model respects the principle of dual diminishing transfer, then $u(x)=x$ for all $x$ in $\mathbb{R}_{+}$, up to an increasing affine transformation.

Proof: See the appendix.

Theorem 4 If a decision maker who behaves in accordance with the RDEU model respects the principle of diminishing transfer, then $f(p)=p$ for all $p$ in $] 0,1[$.

Proof: See the appendix.

It immediately follows from Theorems 3 and 4 that if a decision maker who behaves in accordance with the RDEU model respects the principle of diminishing transfer and the principle of dual diminishing transfer, then his social welfare function reduces to $W(X)=\frac{1}{n} \sum_{i=1}^{n} x_{i}$. This result motivates the introduction of the principle of strong diminishing transfer and the following results.

Theorem 5 If a decision maker who behaves in accordance with the RDEU model respects the principle of strong diminishing transfer, then $f^{\prime \prime \prime}(p) \geq 0$ for all $p$ in $] 0,1\left[\right.$ and $u^{\prime \prime \prime}(x) \geq 0$ for all $x$ in $\mathbb{R}_{++}$.

Proof: See the appendix.

Remark: The converse of Theorem 5 does not hold, as shown by the following example.

\section{Example}

Let us choose the utility function $u(x)=x^{2}$ for all $x$ in $\mathbb{R}_{+}$, and the frequency distortion function $f(p)=p^{2}$ for all $p$ in $[0,1]$. Obviously, $u^{\prime \prime \prime}(x) \geq 0$ for all $x$ in $\mathbb{R}_{+}$and $f^{\prime \prime \prime}(p) \geq 0$ for all $p$ in $[0,1]$. Note, furthermore, that $u(x)=f(x)$ for all $x$ in $[0,1]$.

Now, define $Z_{1}$ and $Z_{2}$ as in Definition 4 , choosing $n=8, i_{1}=1, i_{2}=3, i_{3}=5, i_{4}=7, \varepsilon=\frac{1}{n}$ and: $0<z_{i_{1}}=z=\frac{n-i_{4}}{n}<z_{i_{2}}=z+\alpha=\frac{n-i_{3}}{n}<z_{i_{3}}=y=\frac{n-i_{2}}{n}<z_{i_{4}}=y+\alpha=\frac{n-i_{1}}{n}<1$. Thus, $\alpha=\frac{2}{n}$. With these requirements, the expression $\delta=W\left(u\left(Z_{1}\right)\right)-W\left(u\left(Z_{2}\right)\right)$ becomes:

$$
\begin{aligned}
\delta= & {\left[u\left(z_{i_{1}}+\varepsilon\right)-u\left(z_{i_{1}}\right)\right]\left[u\left(z_{i_{4}}+\varepsilon\right)-u\left(z_{i_{4}}\right)\right]-\left[u\left(z_{i_{2}}\right)-u\left(z_{i_{2}}-\varepsilon\right)\right]\left[u\left(z_{i_{3}}+\varepsilon\right)-u\left(z_{i_{3}}\right)\right] } \\
& -\left[u\left(z_{i_{3}}+\varepsilon\right)-u\left(z_{i_{3}}\right)\right]\left[u\left(z_{i_{3}}+\varepsilon\right)-u\left(z_{i_{2}}\right)\right]+\left[u\left(z_{i_{4}}\right)-u\left(z_{i_{4}}-\varepsilon\right)\right]\left[u\left(z_{i_{1}}+\varepsilon\right)-u\left(z_{i_{1}}\right)\right] .
\end{aligned}
$$


Therefore,

$$
\begin{aligned}
\delta & =\left[u\left(z_{i_{1}}+\varepsilon\right)-u\left(z_{i_{1}}\right)\right]\left[u\left(z_{i_{4}}+\varepsilon\right)-u\left(z_{i_{4}}-\varepsilon\right)\right]-\left[u\left(z_{i_{3}}+\varepsilon\right)-u\left(z_{i_{3}}\right)\right]\left[u\left(z_{i_{2}}+\varepsilon\right)-u\left(z_{i_{2}}-\varepsilon\right)\right] \\
& =[u(z+\varepsilon)-u(z)][u(y+\alpha+\varepsilon)-u(y+\alpha-\varepsilon)]-[u(y+\varepsilon)-u(y)][u(z+\alpha+\varepsilon)-u(z+\alpha-\varepsilon)] \\
& =\left[(z+\varepsilon)^{2}-z^{2}\right]\left[(y+\alpha+\varepsilon)^{2}-(y+\alpha-\varepsilon)^{2}\right]-\left[(y+\varepsilon)^{2}-y^{2}\right]\left[(z+\alpha+\varepsilon)^{2}-(z+\alpha-\varepsilon)^{2}\right] \\
& =4 \varepsilon^{2}(y-z)(\varepsilon-2 \alpha)<0 .
\end{aligned}
$$

Hence, there are $f$ and $u$ with $f^{\prime \prime \prime} \geq 0$ and $u^{\prime \prime \prime} \geq 0$ such that a decision maker who behaves in accordance with the RDEU model does not respect the principle of strong diminishing transfer.

Theorem 6 For a decision maker who behaves in accordance with the RDEU model, the two following propositions are equivalent:

(i) the decision maker respects the principle of transfer and the principle of strong diminishing transfer,

(ii) $f^{\prime \prime}(p) \geq 0, u^{\prime \prime}(x) \leq 0, f^{\prime \prime \prime}(p) \geq 0$ and $u^{\prime \prime \prime}(x) \geq 0$ for all $p$ in $] 0,1\left[\right.$ and all $x$ in $\mathbb{R}_{++}$.

Proof: See the appendix.

It may be of interest to note that a counterpart of Theorem 6 exists when the decision maker doesn't like any Pigou-Dalton transfer. Of course, such an assumption doesn't really make sense in the framework of inequality measurement. On the other hand, it may be of interest in the context of a decision maker's attitude towards risk. In this case, we say that any decision maker who respects the principle of transfer is risk averse (i.e., he respects second order stochastic dominance), whereas a decision maker who doesn't like any Pigou-Dalton transfer is a risk lover. We then have the following result.

Theorem 7 For a decision maker who behaves in accordance with the RDEU model, the two following propositions are equivalent:

(i) the decision maker is a risk lover and respects the principle of strong diminishing transfer,

(ii) $f^{\prime \prime}(p) \leq 0, u^{\prime \prime}(x) \geq 0, f^{\prime \prime \prime}(p) \geq 0$ and $u^{\prime \prime \prime}(x) \geq 0$ for all $p$ in $] 0,1\left[\right.$ and all $x$ in $\mathbb{R}_{++}$.

Proof: Similar to the proof of Theorem 6.

As we can see, the conditions on the sign of the third derivatives of $u$ and $f$ are the same when the decision maker is risk averse and when he is risk lover. Furthermore, it should be noted that, unlike in Example 1, $u^{\prime \prime}$ and $f^{\prime \prime}$ have opposite signs in Theorems 6 and 7. 


\section{Application : generalized Gini indices and super-generalized Gini indices}

We will now apply these results to the problem of inequality measurement.

Let us consider the large class of inequality indices, introduced by Yaari (1988). These indices are defined as ${ }^{3}$ :

$$
I_{G G}(X)=1-\frac{W(X)}{\bar{X}} .
$$

Applying Theorem 2, it follows that:

Theorem 8 A Yaari inequality index satisfies the principle of dual diminishing transfer if and only if $f^{\prime \prime \prime}(p) \geq 0$ for all $\left.p \in\right] 0,1[$.

Donaldson and Weymark (1980) and Bossert (1990) define the sub-class of Yaari indices which satisfy an aggregation axiom. These indices, known as S-Gini indices, are defined as follows ${ }^{4}$ :

$$
I_{S G}(X)=1-\frac{\sum_{i=1}^{n}\left[\left(\frac{n-i+1}{n}\right)^{\delta}-\left(\frac{n-i}{n}\right)^{\delta}\right] x_{i}}{\bar{X}}
$$

with $\delta \geq 1$. These indices are based on the following social welfare function:

$$
W_{S G}(X)=\sum_{i=1}^{n}\left[f\left(\frac{n-i+1}{n}\right)-f\left(\frac{n-i}{n}\right)\right] x_{i} .
$$

with $f(p)=p^{\delta}$. Note that for $\delta=2, I_{S G}$ is nothing but the Gini index. Furthermore, the decision maker is more inequality averse the higher $\delta$ is.

Applying Theorem 2 again, we then obtain:

Theorem 9 An S-Gini index respects the principle of dual diminishing transfer if and only if $\delta \geq 2$.

Hence, the Gini index corresponds to the lowest value of $\delta$ for which $I_{S G}$ respects the principle of dual diminishing transfer.

One may ask if it is possible to define an inequality index which respects the principle of diminishing transfer and the principle of dual diminishing transfer. We have no answer to this question. Nevertheless, the very general inequality index (let us call it a super-generalized Gini index),

$$
I_{S S G}(X)=1-\frac{u^{-1}\left(\sum_{i=1}^{n}\left[f\left(\frac{n-i+1}{n}\right)-f\left(\frac{n-i}{n}\right)\right] u\left(x_{i}\right)\right)}{\bar{X}} .
$$

\footnotetext{
${ }^{3}$ We will consider, without loss of generality, relative Yaari indices.

${ }^{4}$ Indeed, these indices are conceived by Donaldson and Weymark (1980) and Bossert (1990) as an extension of Weymark's indices (1981). But retrospectively these indices appear as a particular case of Yaari indices.
} 
considered by Ebert (1988) and Chateauneuf (1996) is based on a RDEU-like social welfare function, with a utility function $u$ and a frequency transformation function $f$. Applying Theorem 3 , it immediately follows that a super-generalized Gini index respects the principle of transfer and the principle of strong diminishing transfer if and only if $u^{\prime \prime}(x) \leq 0, u^{\prime \prime \prime}(x) \geq 0$ for all $x$ in $\mathbb{R}_{++}$and $f^{\prime \prime}(p) \geq 0, f^{\prime \prime \prime}(p) \geq 0$ for all $p$ in $] 0,1[$. This may be seen as an argument in favor of this particular class of indices.

\section{$5 \quad$ Appendix}

The proofs rely on the following lemma:

Lemma Let $g$ be a continuous real function on $I=[0, a], a \in \mathbb{R}_{+}\left(\right.$resp. $\left.I=\mathbb{R}_{+}\right)$three times differentiable on $] 0, a\left[\right.$ (resp. on $\mathbb{R}_{++}$). Then $g^{\prime \prime \prime}(x) \geq 0$ for all $x$ in $] 0, a\left[\right.$ (resp: in $\mathbb{R}_{++}$) if and only if $\Delta_{3}\left(x ; a_{1}, a_{2}, a_{3}\right) \geq 0$ for all $x, a_{1}, a_{2}, a_{3}$ in $I$ such that $x+a_{1}+a_{2}+a_{3}$ belongs to $I$, where $\Delta_{3}\left(x ; a_{1}, a_{2}, a_{3}\right)$ is defined by:

$$
\begin{aligned}
\Delta_{3}\left(x ; a_{1}, a_{2}, a_{3}\right)= & g\left(x+a_{1}+a_{2}+a_{3}\right)-g\left(x+a_{1}+a_{2}\right)-g\left(x+a_{1}+a_{3}\right)-g\left(x+a_{2}+a_{3}\right) \\
& +g\left(x+a_{1}\right)+g\left(x+a_{2}\right)+g\left(x+a_{3}\right)-g(x) .
\end{aligned}
$$

Such a result is proved in Choquet (1954) (p. 149) in the case where $I=\mathbb{R}_{+}$and $g$ is three times differentiable on $I=\mathbb{R}_{+}$, from which can be readily deduced the slight generalization above.

\section{Proof of Theorem 1}

$\underline{(i i) \Longrightarrow(i)}$

A decision maker who behaves in accordance respects the principle of diminishing transfer if and only if for all $X_{1}$ and $X_{2}$ as in Definition 2, $U\left(X_{1}\right)-U\left(X_{2}\right) \geq 0$. A simple computation leads to:

$$
\begin{aligned}
U\left(X_{1}\right)-U\left(X_{2}\right)= & u\left(x_{i_{4}}\right)-u\left(x_{i_{4}}-\varepsilon\right)-u\left(x_{i_{3}}+\varepsilon\right)-u\left(x_{i_{2}}\right) \\
& +u\left(x_{i_{3}}\right)+u\left(x_{i_{2}}-\varepsilon\right)+u\left(x_{i_{1}}+\varepsilon\right)-u\left(x_{i_{1}}\right) .
\end{aligned}
$$

Let $x=x_{i_{1}}, a_{1}=\varepsilon, a_{2}=x_{i_{2}}-x_{i_{1}}-\varepsilon, a_{3}=x_{i_{3}}-x_{i_{1}}$. Note that $x, a_{1}, a_{2}$ and $a_{3}$ belong to $\mathbb{R}_{+}$. One easily checks that:

$$
U\left(X_{1}\right)-U\left(X_{2}\right)=\Delta_{3}\left(x ; a_{1}, a_{2}, a_{3}\right)
$$


Hence, from the Lemma, if $u^{\prime \prime \prime}(x) \geq 0$ for all $x$ in $\mathbb{R}_{++}$then $U\left(X_{1}\right) \geq U\left(X_{2}\right)$.

$\underline{(i) \Longrightarrow(i i)}$

Let $y, x$ be fixed, with $y>x>0$. We have to prove that $u^{\prime \prime}(y) \geq u^{\prime \prime}(x)$.

Let $x_{i_{4}}=y+\alpha, x_{i_{3}}=y, x_{i_{2}}=x+\alpha$ and $x_{i_{1}}=x$, where $\alpha>0$ is arbitrarily chosen such that $x_{i_{3}} \geq x_{i_{2}}$. Divide both sides of equation (1) by $\varepsilon>0$, and let $\varepsilon$ tend to 0 . One then obtains:

$$
u^{\prime}(y+\alpha)-u^{\prime}(y) \geq u^{\prime}(x+\alpha)-u^{\prime}(x)
$$

Divide both sides of this inequality by $\alpha$ and let $\alpha$ tend to 0 . It follows that $u^{\prime \prime}(y) \geq u^{\prime \prime}(x)$.

\section{Proof of Theorem 2}

$\underline{(i i) \Longrightarrow(i)}$

Assume that $f^{\prime \prime \prime}(p) \geq 0$ for all $p$ belonging to $] 0,1\left[\right.$ and let us show that $W\left(Y_{1}\right) \geq W\left(Y_{2}\right)$ with $Y_{1}$ and $Y_{2}$ as in Definition 3. A simple computation gives:

$$
\begin{aligned}
W\left(Y_{1}\right)-W\left(Y_{2}\right)= & \left(y_{i_{1}}+\varepsilon\right) \Psi\left(\frac{i_{1}}{n}\right)+\left(y_{i_{2}}-\varepsilon\right) \Psi\left(\frac{i_{2}}{n}\right)+y_{i_{3}} \Psi\left(\frac{i_{3}}{n}\right)+y_{i_{4}} \Psi\left(\frac{i_{4}}{n}\right) \\
& -y_{i_{1}} \Psi\left(\frac{i_{1}}{n}\right)-y_{i_{2}} \Psi\left(\frac{i_{2}}{n}\right)-\left(y_{i_{3}}+\varepsilon\right) \Psi\left(\frac{i_{3}}{n}\right)-\left(y_{i_{4}}-\varepsilon\right) \Psi\left(\frac{i_{4}}{n}\right) .
\end{aligned}
$$

Hence, $W\left(Y_{1}\right)-W\left(Y_{2}\right)=\varepsilon d$, with:

$$
\begin{aligned}
d= & f\left(\frac{n-i_{1}+1}{n}\right)-f\left(\frac{n-i_{1}}{n}\right)-f\left(\frac{n-i_{2}+1}{n}\right)-f\left(\frac{n-i_{3}+1}{n}\right) \\
& +f\left(\frac{n-i_{2}}{n}\right)+f\left(\frac{n-i_{3}}{n}\right)+f\left(\frac{n-i_{4}+1}{n}\right)-f\left(\frac{n-i_{4}}{n}\right) .
\end{aligned}
$$

Let $x=\frac{n-i_{4}}{n}, a_{1}=\frac{1}{n}, a_{2}=\frac{i_{4}-i_{3}}{n}, a_{3}=\frac{i_{4}-i_{2}}{n} ; x$ and $a_{i}$ belonging to $[0,1]$ and $d=$ $\Delta_{3}\left(x ; a_{1}, a_{2}, a_{3}\right)$. Hence, from the Lemma, $d \geq 0$, which gives the desired result.

$\underline{(i) \Longrightarrow(i i)}$

From continuity of $f^{\prime \prime}$ on $] 0,1\left[\right.$, it is enough to prove that for any given $p_{1}, p_{2}$ belonging to $\mathbb{Q} \cap] 0,1\left[\right.$ with $p_{2}>p_{1}$, we obtain $f^{\prime \prime}\left(p_{2}\right) \geq f^{\prime \prime}\left(p_{1}\right)$.

Let us choose a sufficiently small $q$ belonging to the set $\mathbb{Q}_{++}$of positive rationals, such that $p_{1}+q<p_{2}$ and $p_{2}+q<1$ and let $n$ in the set $\mathbb{N}^{*}$ of positive integers be chosen such that $p_{2}=\frac{m_{2}}{n}, p_{1}=\frac{m_{1}}{n}, q=\frac{m}{n}$. 
Consider now $Y_{1}$ and $Y_{2}$ of size $k n$, with $k$ belonging to $\mathbb{N}^{*}$, defined as in definition 3 and with $i_{1}=k n-k\left(m+m_{2}\right), i_{2}=k n-k m_{2}, i_{3}=k n-k\left(m_{1}+m\right), i_{4}=k n-k m_{1} . W\left(Y_{1}\right)-W\left(Y_{2}\right) \geq 0$ therefore implies:

$$
\begin{aligned}
& {\left[f\left(p_{2}+q+\frac{1}{k n}\right)-f\left(p_{2}+q\right)\right]-\left[f\left(p_{2}+\frac{1}{k n}\right)-f\left(p_{2}\right)\right] } \\
\geq & {\left[f\left(p_{1}+q+\frac{1}{k n}\right)-f\left(p_{1}+q\right)\right]-\left[f\left(p_{1}+\frac{1}{k n}\right)-f\left(p_{1}\right)\right] . }
\end{aligned}
$$

Divide by $\frac{1}{k n}$ and let $k$ tend to $+\infty$. It follows that:

$$
f^{\prime}\left(p_{2}+q\right)-f^{\prime}\left(p_{2}\right) \geq f^{\prime}\left(p_{1}+q\right)-f^{\prime}\left(p_{1}\right) .
$$

Dividing now by $q$ and letting $q$ tending to 0 gives the desired result: $f^{\prime \prime}\left(p_{2}\right) \geq f^{\prime \prime}\left(p_{1}\right)$.

\section{Proof of Theorem 3}

$\underline{\text { We first prove that } u^{\prime \prime}(x) \geq 0 \text { for all } x \text { in } \mathbb{R}_{++}}$

Let $\left(1-\frac{p}{q}\right)$ belonging to $\left.\mathbb{Q} \cap\right] 0,1\left[\right.$ be such that $f^{\prime}\left(1-\frac{p}{q}\right)>0$. Consider $Y=\left(y_{1}, \ldots, y_{n}\right)$, and $Y_{1}$ and $Y_{2}$ as in Definition 3. We assume that $n=q r m$, where $r>3$ and $m$ are arbitrarily chosen in $\mathbb{N}^{*}$. Let $i_{1}=p(r-3) m, i_{2}=p(r-2) m, i_{3}=p(r-1) m, i_{4}=p r m$. Finally, let $y_{i_{1}}$, $y_{i_{2}}, y_{i_{3}}, y_{i_{4}}$ and $\alpha>0$ be arbitrarily chosen such that $0<y_{i_{1}}<y_{i_{2}}=y_{i_{1}}+\alpha<y_{i_{3}}<y_{i_{4}}$.

Then, $W\left(u\left(Y_{1}\right)\right)-W\left(u\left(Y_{2}\right)\right) \geq 0$ with $\varepsilon$ sufficiently small implies:

$$
\begin{aligned}
& {\left[u\left(y_{i_{4}}\right)-u\left(y_{i_{4}}-\varepsilon\right)\right] \Psi\left(\frac{i_{4}}{n}\right)-\left[u\left(y_{i_{3}}+\varepsilon\right)-u\left(y_{i_{3}}\right)\right] \Psi\left(\frac{i_{3}}{n}\right) } \\
\geq & {\left[u\left(y_{i_{1}}+\alpha\right)-u\left(y_{i_{1}}+\alpha-\varepsilon\right)\right] \Psi\left(\frac{i_{2}}{n}\right)-\left[u\left(y_{i_{1}}+\varepsilon\right)-u\left(y_{i_{1}}\right)\right] \Psi\left(\frac{i_{1}}{n}\right) . }
\end{aligned}
$$

First, let $r$ be constant. Divide expression (2) by $\frac{1}{q r m}$ and let $m$ tend to $+\infty$. It follows that:

$$
\begin{aligned}
& {\left[u\left(y_{i_{4}}\right)-u\left(y_{i_{4}}-\varepsilon\right)\right] f^{\prime}\left(1-\frac{p}{q}\right)-\left[u\left(y_{i_{3}}+\varepsilon\right)-u\left(y_{i_{3}}\right)\right] f^{\prime}\left(1-\frac{p}{q}+\frac{p}{q r}\right) } \\
\geq & {\left[u\left(y_{i_{1}}+\alpha\right)-u\left(y_{i_{1}}+\alpha-\varepsilon\right)\right] f^{\prime}\left(1-\frac{p}{q}+2 \frac{p}{q r}\right)-\left[u\left(y_{i_{1}}+\varepsilon\right)-u\left(y_{i_{1}}\right)\right] f^{\prime}\left(1-\frac{p}{q}+3 \frac{p}{q r}\right) . }
\end{aligned}
$$

Now, let $r$ tend to $+\infty$. It follows that: $f^{\prime}\left(1-\frac{p}{q}\right) \delta \geq 0$, where

$$
\begin{aligned}
\delta= & {\left[u\left(y_{i_{4}}\right)-u\left(y_{i_{4}}-\varepsilon\right)\right]-\left[u\left(y_{i_{3}}+\varepsilon\right)-u\left(y_{i_{3}}\right)\right] } \\
& -\left[u\left(y_{i_{1}}+\alpha\right)-u\left(y_{i_{1}}+\alpha-\varepsilon\right)\right]+\left[u\left(y_{i_{1}}+\varepsilon\right)-u\left(y_{i_{1}}\right)\right] .
\end{aligned}
$$


Hence, $\delta \geq 0$. Now, divide $\delta$ by $\varepsilon>0$ and let $\varepsilon$ tend to 0 . One obtains:

$$
u^{\prime}\left(y_{i_{4}}\right)-u^{\prime}\left(y_{i_{3}}\right) \geq u^{\prime}\left(y_{i_{1}}+\alpha\right)-u^{\prime}\left(y_{i_{1}}\right) .
$$

Finally, let $\alpha$ tend to 0 . It follows that:

$$
u^{\prime}\left(y_{i_{4}}\right)-u^{\prime}\left(y_{i_{3}}\right) \geq 0
$$

for all $0<y_{i_{3}}<y_{i_{4}}$. Hence, $u^{\prime \prime}(x) \geq 0$ for all $x$ in $\mathbb{R}_{++}$.

$\underline{\text { We now prove that } u^{\prime \prime}(x) \leq 0 \text { for all } x \text { in } \mathbb{R}_{++}}$

With the same notation as in the first part of the proof, let $y_{i_{1}}, y_{i_{2}}, y_{i_{3}}, y_{i_{4}}$ and $\beta>0$ be arbitrarily chosen such that $0<y_{i_{1}}<y_{i_{2}}<y_{i_{3}}=y_{i_{4}}-\beta<y_{i_{4}}$.

Then, $W\left(u\left(Y_{1}\right)\right)-W\left(u\left(Y_{2}\right)\right) \geq 0$ with $\varepsilon$ sufficiently small implies:

$$
\begin{aligned}
& {\left[u\left(y_{i_{4}}\right)-u\left(y_{i_{4}}-\varepsilon\right)\right] \Psi\left(\frac{i_{4}}{n}\right)-\left[u\left(y_{i_{4}}-\beta+\varepsilon\right)-u\left(y_{i_{4}}-\beta\right)\right] \Psi\left(\frac{i_{3}}{n}\right) } \\
\geq & {\left[u\left(y_{i_{2}}\right)-u\left(y_{i_{2}}-\varepsilon\right)\right] \Psi\left(\frac{i_{2}}{n}\right)-\left[u\left(y_{i_{1}}+\varepsilon\right)-u\left(y_{i_{1}}\right)\right] \Psi\left(\frac{i_{1}}{n}\right) . }
\end{aligned}
$$

The same argument as in the first part of the proof leads to:

$$
u^{\prime}\left(y_{i_{4}}\right)-u^{\prime}\left(y_{i_{4}}-\beta\right) \geq u^{\prime}\left(y_{i_{2}}\right)-u^{\prime}\left(y_{i_{1}}\right) .
$$

Now, let $\beta$ tend to 0 . It follows that:

$$
u^{\prime}\left(y_{i_{2}}\right)-u^{\prime}\left(y_{i_{1}}\right) \leq 0
$$

for all $0<y_{i_{1}}<y_{i_{2}}$. Hence, $u^{\prime \prime}(x) \leq 0$ for all $x$ in $\mathbb{R}_{++}$.

Hence, $u^{\prime \prime}(x) \geq 0$ and $u^{\prime \prime}(x) \leq 0$ for all $x$ in $\mathbb{R}_{++}$. Therefore, $u$ is affine on $\mathbb{R}_{++}$. By continuity of $u$ on $\mathbb{R}_{+}$, this implies that $u$ is also affine on $\mathbb{R}_{+}$. Hence, $u(x)=x$ on $\mathbb{R}_{+}$, up to a positive affine transformation.

\section{Proof of Theorem 4}

We first prove that $f^{\prime \prime}(p) \leq 0$ for all $p$ in $] 0,1[$ 
Let $\frac{a^{\prime}}{b^{\prime}}<\frac{a}{b}$ belonging to $\left.\mathbb{Q} \cap\right] 0,1\left[\right.$, and $x>0$ be such that $u^{\prime}(x)>0$. Consider $X=\left(x_{1}, \ldots, x_{n}\right)$ and $X_{1}, X_{2}$ as in Definition 2.

We assume that $n=b b^{\prime} r m$, where $r>2$ and $m$ are arbitrarily chosen in $\mathbb{N}^{*}$. Let $i_{1}=$ $(b-a) b^{\prime}(r-2) m, i_{2}=(b-a) b^{\prime}(r-1) m, i_{3}=(b-a) b^{\prime} r m$ and $i_{4}=b\left(b^{\prime}-a^{\prime}\right) r m$. It is immediate that $i_{1}<i_{2}<i_{3}<i_{4}$. Finally, let $\alpha>0$ be arbitrarily chosen, and let $x_{i_{1}}, x_{i_{2}}, x_{i_{3}}$, $x_{i_{4}}$ be such that $x_{i_{1}}=x<x_{i_{2}}=x_{i_{1}}+\alpha<x_{i_{3}}=x_{i_{1}}+2 \alpha<x_{i_{4}}=x_{i_{1}}+3 \alpha$.

Then, $W\left(u\left(X_{1}\right)\right)-W\left(u\left(X_{2}\right)\right) \geq 0$ with $\varepsilon$ sufficiently small implies:

$$
\begin{aligned}
& {\left[u\left(x_{i_{1}}+3 \alpha\right)-u\left(x_{i_{1}}+3 \alpha-\varepsilon\right)\right] \Psi\left(\frac{i_{4}}{n}\right)-\left[u\left(x_{i_{1}}+2 \alpha+\varepsilon\right)-u\left(x_{i_{1}}+2 \alpha\right)\right] \Psi\left(\frac{i_{3}}{n}\right) } \\
\geq & {\left[u\left(x_{i_{1}}+\alpha\right)-u\left(x_{i_{1}}+\alpha-\varepsilon\right)\right] \Psi\left(\frac{i_{2}}{n}\right)-\left[u\left(x_{i_{1}}+\varepsilon\right)-u\left(x_{i_{1}}\right)\right] \Psi\left(\frac{i_{1}}{n}\right) . }
\end{aligned}
$$

Divide (3) by $\varepsilon$ and let $\varepsilon$ tend to 0 . It follows that:

$$
u^{\prime}\left(x_{i_{1}}+3 \alpha\right) \Psi\left(\frac{i_{4}}{n}\right)-u^{\prime}\left(x_{i_{1}}+2 \alpha\right) \Psi\left(\frac{i_{3}}{n}\right) \geq u^{\prime}\left(x_{i_{1}}+\alpha\right) \Psi\left(\frac{i_{2}}{n}\right)-u^{\prime}\left(x_{i_{1}}\right) \Psi\left(\frac{i_{1}}{n}\right) .
$$

Now let $\alpha$ tend to 0 . We obtain:

$$
u^{\prime}\left(x_{i_{1}}\right)\left[\Psi\left(\frac{i_{4}}{n}\right)-\Psi\left(\frac{i_{3}}{n}\right)-\Psi\left(\frac{i_{2}}{n}\right)+\Psi\left(\frac{i_{1}}{n}\right)\right] \geq 0 .
$$

Since $u^{\prime}\left(x_{i_{1}}\right)>0$, this implies that $\left[\Psi\left(\frac{i_{4}}{n}\right)-\Psi\left(\frac{i_{3}}{n}\right)-\Psi\left(\frac{i_{2}}{n}\right)+\Psi\left(\frac{i_{1}}{n}\right)\right] \geq 0$. Hence:

$$
\begin{aligned}
& {\left[f\left(\frac{a^{\prime}}{b^{\prime}}+\frac{1}{b b^{\prime} r m}\right)-f\left(\frac{a^{\prime}}{b^{\prime}}\right)\right] } \\
+ & {\left[f\left(1-\frac{(b-a)(r-2)}{b r}+\frac{1}{b b^{\prime} r m}\right)-f\left(1-\frac{(b-a)(r-2)}{b r}\right)\right] } \\
\geq & {\left[f\left(1-\frac{(b-a)(r-1)}{b r}+\frac{1}{b b^{\prime} r m}\right)-f\left(1-\frac{(b-a)(r-1)}{b r}\right)\right] } \\
& +\left[f\left(\frac{a}{b}+\frac{1}{b b^{\prime} r m}\right)-f\left(\frac{a}{b}\right)\right]
\end{aligned}
$$

First, let $r$ be constant. Divide (4) by $\frac{1}{b b^{\prime} r m}$ and let $m$ tend to $+\infty$. It follows that:

$$
f^{\prime}\left(\frac{a^{\prime}}{b^{\prime}}\right)+f^{\prime}\left(1-\frac{(b-a)(r-2)}{b r}\right)-f^{\prime}\left(1-\frac{(b-a)(r-1)}{b r}\right)-f^{\prime}\left(\frac{a}{b}\right) \geq 0 .
$$

Now, let $r$ tend to $+\infty$. We then have:

$$
f^{\prime}\left(\frac{a^{\prime}}{b^{\prime}}\right) \geq f^{\prime}\left(\frac{a}{b}\right)
$$

for all $0<\frac{a^{\prime}}{b^{\prime}}<\frac{a}{b}$. Hence, $f^{\prime \prime}(p) \leq 0$ for all $p$ in $\left.\mathbb{Q} \cap\right] 0,1[$, and by continuity on $] 0,1[$. 
We now prove that $f^{\prime \prime}(p) \geq 0$ for all $p$ in $] 0,1[$

Let $\frac{a^{\prime}}{b^{\prime}}<\frac{a}{b}$ belonging to $\left.\mathbb{Q} \cap\right] 0,1\left[\right.$, and $x>0$ be such that $u^{\prime}(x)>0$. Consider $X=\left(x_{1}, \ldots, x_{n}\right)$ and $X_{1}, X_{2}$ as in Definition 2.

We assume that $n=b b^{\prime} r m$, where $r$ and $m$ are arbitrarily chosen in $\mathbb{N}^{*}$, such that $1-\frac{a^{\prime}}{b^{\prime}} \leq \frac{r}{r+2}$. Let $i_{1}=(b-a) b^{\prime} r m, i_{2}=b\left(b^{\prime}-a^{\prime}\right) r m, i_{3}=b\left(b^{\prime}-a^{\prime}\right)(r+1) m$ and $i_{4}=b\left(b^{\prime}-a^{\prime}\right)(r+2) m$. It is immediate that $i_{1}<i_{2}<i_{3}<i_{4} \leq n$. Finally, let $\alpha>0$ be arbitrarily chosen, and let $x_{i_{1}}$, $x_{i_{2}}, x_{i_{3}}, x_{i_{4}}$ be such that $x_{i_{1}}=x<x_{i_{2}}=x_{i_{1}}+\alpha<x_{i_{3}}=x_{i_{1}}+2 \alpha<x_{i_{4}}=x_{i_{1}}+3 \alpha$.

As in the first part of the proof, $W\left(u\left(X_{1}\right)\right)-W\left(u\left(X_{2}\right)\right) \geq 0$ with $\varepsilon$ sufficiently small implies that

$$
\left[\Psi\left(\frac{i_{4}}{n}\right)-\Psi\left(\frac{i_{3}}{n}\right)-\Psi\left(\frac{i_{2}}{n}\right)+\Psi\left(\frac{i_{1}}{n}\right)\right] \geq 0
$$

Hence:

$$
\begin{aligned}
& {\left[f\left(1-\frac{\left(b^{\prime}-a^{\prime}\right)(r+2)}{b^{\prime} r}+\frac{1}{b b^{\prime} r m}\right)-f\left(1-\frac{\left(b^{\prime}-a^{\prime}\right)(r+2)}{b^{\prime} r}\right)\right] } \\
- & {\left[f\left(1-\frac{\left(b^{\prime}-a^{\prime}\right)(r+1)}{b^{\prime} r}+\frac{1}{b b^{\prime} r m}\right)-f\left(1-\frac{\left(b^{\prime}-a^{\prime}\right)(r+1)}{b^{\prime} r}\right)\right] } \\
- & {\left[f\left(\frac{a^{\prime}}{b^{\prime}}+\frac{1}{b b^{\prime} r m}\right)-f\left(\frac{a^{\prime}}{b^{\prime}}\right)\right]+\left[f\left(\frac{a}{b}+\frac{1}{b b^{\prime} r m}\right)-f\left(\frac{a}{b}\right)\right] \geq 0 }
\end{aligned}
$$

First, let $r$ be constant. Divide (5) by $\frac{1}{b b^{\prime} r m}$ and let $m$ tend to $+\infty$. It then follows that

$$
f^{\prime}\left(\frac{a}{b}\right)+f^{\prime}\left(1-\frac{\left(b^{\prime}-a^{\prime}\right)(r+2)}{b^{\prime} r}\right)-f^{\prime}\left(1-\frac{\left(b^{\prime}-a^{\prime}\right)(r+1)}{b^{\prime} r}\right) \geq f^{\prime}\left(\frac{a^{\prime}}{b^{\prime}}\right)
$$

Now, let $r$ tend to $+\infty$. We then have:

$$
f^{\prime}\left(\frac{a}{b}\right) \geq f^{\prime}\left(\frac{a^{\prime}}{b^{\prime}}\right)
$$

for all $0<\frac{a^{\prime}}{b^{\prime}}<\frac{a}{b}$. Hence $f^{\prime \prime}(p) \geq 0$ for all $p$ in $\left.\mathbb{Q} \cap\right] 0,1[$, and by continuity on $] 0,1[$.

We finally have $f^{\prime \prime}(p)=0$ for all $p$ in $] 0,1[$. Since $f(0)=0$ and $f(1)=1$, this implies $f(p)=p$.

\section{Proof of Theorem 5}

Consider $x$ belonging to $\mathbb{R}_{++}$and let us show that $u^{\prime \prime \prime}(x) \geq 0$

Let $1-\frac{p}{q}$ belonging to $\left.\mathbb{Q} \cap\right] 0,1\left[\right.$ be such that $f^{\prime}\left(1-\frac{p}{q}\right)>0$. Consider $Z_{1}$ and $Z_{2}$ defined as in definition 4 of size $n=q r m$, where $r$ and $m$ are arbitrarily fixed in $\mathbb{N}^{*}$, and $i_{1}=p r m$, 
$i_{2}=p(r+1) m, i_{3}=p(r+2) m, i_{4}=p(r+3) m$, and $z_{i_{1}}=z, z_{i_{2}}=z+\alpha, z_{i_{3}}=y$, $z_{i_{4}}=y+\alpha$, where $y>x$ and $\alpha>0$ are arbitrarily chosen.

$W\left(u\left(Z_{1}\right)\right)-W\left(u\left(Z_{2}\right)\right) \geq 0$ with $\varepsilon>0$ sufficiently small then implies:

$$
\begin{aligned}
& {[u(y+\alpha)-u(y+\alpha-\varepsilon)] \Psi\left(\frac{i_{4}}{n}\right)-[u(y+\varepsilon)-u(y)] \Psi\left(\frac{i_{3}}{n}\right) } \\
\geq & {[u(z+\alpha)-u(z+\alpha-\varepsilon)] \Psi\left(\frac{i_{2}}{n}\right)-[u(z+\varepsilon)-u(z)] \Psi\left(\frac{i_{1}}{n}\right) . }
\end{aligned}
$$

First, let $r$ be fixed. Dividing by $\frac{1}{q r m}$, and letting $m$ tend to $+\infty$, it follows that

$$
\begin{aligned}
& {[u(y+\alpha)-u(y+\alpha-\varepsilon)] f^{\prime}\left(1-\frac{p}{q}-3 \frac{p}{q r}\right)-[u(y+\varepsilon)-u(y)] f^{\prime}\left(1-\frac{p}{q}-2 \frac{p}{q r}\right) } \\
\geq & {[u(z+\alpha)-u(z+\alpha-\varepsilon)] f^{\prime}\left(1-\frac{p}{q}-\frac{p}{q r}\right)-[u(z+\varepsilon)-u(z)] f^{\prime}\left(1-\frac{p}{q}\right) . }
\end{aligned}
$$

Now, let $r$ tend to $+\infty$, it follows that $f^{\prime}\left(1-\frac{p}{q}\right) \delta \geq 0$, where:

$\delta=[u(y+\alpha)-u(y+\alpha-\varepsilon)]-[u(y+\varepsilon)-u(y)]-[u(z+\alpha)-u(z+\alpha-\varepsilon)]+u(z+\varepsilon)-u(z)$.

Hence, successively dividing by $\varepsilon>0, \varepsilon$ tending to 0 , and by $\alpha>0, \alpha$ tending to 0 , leads us to:

$$
u^{\prime \prime}(y) \geq u^{\prime \prime}(z)
$$

the desired result.

Let us now prove that for given $p_{1}, p_{2}$ belonging to $\left.\mathbb{Q} \cap\right] 0,1\left[\right.$ with $p_{2}>p_{1}$, we obtain $f^{\prime \prime}\left(p_{2}\right) \geq f^{\prime \prime}\left(p_{1}\right)$

Define $Y_{1}$ and $Y_{2}$ of size $k n$ as in the proof of the necessary part of Theorem 2, but with $y_{i_{2}}-y_{i_{1}}=y_{i_{4}}-y_{i_{3}}$ as in definition 4 .

$W\left(u\left(Y_{1}\right)\right)-W\left(u\left(Y_{2}\right)\right) \geq 0$ implies:

$$
\begin{gathered}
{\left[u\left(y_{i_{1}}+\varepsilon\right)-u\left(y_{i_{1}}\right)\right] \Psi\left(\frac{i_{1}}{n}\right)-\left[u\left(y_{i_{2}}\right)-u\left(y_{i_{2}}-\varepsilon\right)\right] \Psi\left(\frac{i_{2}}{n}\right)} \\
-\left[u\left(y_{i_{3}}+\varepsilon\right)-u\left(y_{i_{3}}\right)\right] \Psi\left(\frac{i_{3}}{n}\right)+\left[u\left(y_{i_{4}}\right)-u\left(y_{i_{4}}-\varepsilon\right)\right] \Psi\left(\frac{i_{4}}{n}\right) \geq 0 .
\end{gathered}
$$

Divide the left hand side of (6) by $\varepsilon>0$ and let $\varepsilon$ tend to 0 . It follows that

$$
u^{\prime}\left(y_{i_{1}}\right) \Psi\left(\frac{i_{1}}{n}\right)-u^{\prime}\left(y_{i_{2}}\right) \Psi\left(\frac{i_{2}}{n}\right)-u^{\prime}\left(y_{i_{3}}\right) \Psi\left(\frac{i_{3}}{n}\right)+u^{\prime}\left(y_{i_{4}}\right) \Psi\left(\frac{i_{4}}{n}\right) \geq 0
$$

Let $y>0$ be chosen belonging to $\mathbb{R}_{++}$such that $u^{\prime}(y)>0$. Let $y_{i_{1}}, y_{i_{2}}, y_{i_{3}}, y_{i_{4}}$ converge to $y$. Since $u^{\prime}$ is continuous on $\mathbb{R}_{++}$and $u^{\prime}(y)>0$, one obtains:

$$
\Psi\left(\frac{i_{1}}{n}\right)-\Psi\left(\frac{i_{2}}{n}\right)-\Psi\left(\frac{i_{3}}{n}\right)+\Psi\left(\frac{i_{4}}{n}\right) \geq 0
$$


and the proof can be completed as in Theorem 2 .

\section{Proof of Theorem 6}

$\underline{(i) \Longrightarrow(i i)}$

We know from Chew, Karni and Safra (1987) that a decision maker who behaves in accordance with the RDEU model with $u$ and $f$ twice differentiable respects second order stochastic dominance if and only if $u$ is concave and $f$ is convex. But it is well-known - see e.g. Atkinson (1970) - that respecting second order stochastic dominance for distributions with equal means is equivalent to respecting the principle of transfer. Hence, if the decision maker respects the principle of transfer, $f^{\prime \prime}(p) \geq 0$ for all $p$ in $] 0,1\left[\right.$ and $u^{\prime \prime}(x) \leq 0$ for all $x$ in $\mathbb{R}_{++}$.

A direct application of Theorem 5 completes this part of the proof.

$\underline{(i i) \Longrightarrow(i)}$

We know from Chew, Karni and Safra (1987) that if $u$ is concave and $f$ is convex, then the decision maker respects the principle of transfer. Hence, it remains to prove that if $u^{\prime \prime \prime}(x) \geq 0$ for all $x$ in $\mathbb{R}_{++}$and $f^{\prime \prime \prime}(p) \geq 0$ for all $p$ in $] 0,1[$, then the decision maker respects the principle of strong diminishing transfer.

Consider $Z_{1}$ and $Z_{2}$ as in definition 4 . We have to prove that $W\left(u\left(Z_{1}\right)\right)-W\left(u\left(Z_{2}\right)\right) \geq 0$, which reduces to proving that $\delta \geq 0$, where $\delta$ is defined by:

$$
\begin{aligned}
\delta= & {\left[u\left(z_{i_{1}}+\varepsilon\right)-u\left(z_{i_{1}}\right)\right] \Psi\left(\frac{i_{1}}{n}\right)-\left[u\left(z_{i_{2}}\right)-u\left(z_{i_{2}}-\varepsilon\right)\right] \Psi\left(\frac{i_{2}}{n}\right) } \\
& -\left[u\left(z_{i_{3}}+\varepsilon\right)-u\left(z_{i_{3}}\right)\right] \Psi\left(\frac{i_{3}}{n}\right)+\left[u\left(z_{i_{4}}\right)-u\left(z_{i_{4}}-\varepsilon\right)\right] \Psi\left(\frac{i_{4}}{n}\right) .
\end{aligned}
$$

By the symmetry of $\delta$ in $i_{2}$ and $i_{3}$, we may assume without loss of generality that $i_{1}<i_{2} \leq i_{3}<$ $i_{4}$. Hence, $z_{i_{1}}<z_{i_{2}} \leq z_{i_{3}}<z_{i_{4}}$.

Let $a_{j} \equiv u\left(z_{i_{j}}+\varepsilon\right)-u\left(z_{i_{j}}\right)$ for $j$ in $\{1,3\}, a_{j} \equiv u\left(z_{i_{j}}\right)-u\left(z_{i_{j}}-\varepsilon\right)$ for $j$ in $\{2,4\}$, and $b_{j} \equiv \Psi\left(\frac{i_{j}}{n}\right)$ for $j$ in $\{1, \ldots, 4\}$.

Since $u^{\prime \prime \prime}(z) \geq 0$ for all $z$ in $\mathbb{R}_{++}$, we know - see Theorem 1 - that a decision maker who behaves in accordance with the expected utility model and whose utility function is $u$ respects the principle of diminishing transfer. Hence, $a \equiv a_{4}-a_{3}-a_{2}+a_{1} \geq 0$.

Since $f^{\prime \prime \prime}(p) \geq 0$ for all $p$ in $] 0,1$, we deduce from Theorem 2 that a decision maker who behaves in accordance with Yaari's dual model and whose frequency transformation function is $f$ respects the principle of dual diminishing transfer. Hence, $b \equiv b_{4}-b_{3}-b_{2}+b_{1} \geq 0$. 
But $\delta=a_{4} b_{4}-a_{3} b_{3}-a_{2} b_{2}+a_{1} b_{1}$. Hence, $\delta=a_{4} b+b_{1} a+d$ with $d=\left(b_{2}-b_{1}\right)\left(a_{4}-a_{2}\right)+$ $\left(b_{3}-b_{1}\right)\left(a_{4}-a_{3}\right)$.

Nondecreasingness of $u$ and $f$ imply $a_{4} \geq 0, b_{1} \geq 0$. Hence, $a_{4} b+b_{1} a \geq 0$. Convexity of $f$ and concavity of $u$ respectively imply $b_{1} \geq b_{2}, b_{1} \geq b_{3}, a_{2} \geq a_{4}, a_{3} \geq a_{4}$. Hence, $d \geq 0$ and therefore $W\left(u\left(Z_{1}\right)\right)-W\left(u\left(Z_{2}\right)\right) \geq 0$.

\section{References}

Atkinson, A. (1970): "On the measurement of inequality," Journal of Economic Theory, 2, $244-263$.

Bossert, W. (1990): "An axiomatization of the Single-Series Ginis," Journal of Economic Theory, 50, 82-92.

Chateauneuf, A. (1996): "Decreasing Inequality : an Approach through non-additive Models," Cahiers EcoMaths, 96.58, Université Paris I.

Chateauneuf, A., and P.-H. Wilthien (1999): "Third Inverse Stochastic Dominance, Lorenz Curves and Favourable Double Transfers," Cahiers de la MSE, 1999.94, Université de Paris I.

Chew, S., E. Karni, and Z. Safra (1987): "Risk aversion in the theory of expected utility with rank dependent preferences," Journal of Economic Theory, 42, 370-381.

Choquet, G. (1954): “Théorie des capacités," Annales de l'Institut Fourier, V, 131-295.

Davies, J., AND M. Hoy (1994): "The Normative Signifiance of Using Third-Degree Stochastic Dominance in Comparing Income Distribution," Journal of Economic Theory, 64, 520-530.

Donaldson, D., and J. Weymark (1980): "A Single-Parameter Generalization of the Gini Indices of Inequality," Journal of Economic Theory, 22, 67-86.

EBERT, U. (1988): "Measurement of inequality: An attempt at unification and generalization," Social Choice and Welfare, 5, 147-169.

Eeckhoudt, L., C. Gollier, and T. Schneider (1995): "Risk-aversion, prudence and temperance: A unified approach," Economics Letters, 48, 331-336.

KakWani, N. C. (1980): "On a class of poverty measures," Econometrica, 48, 437-446.

Kimball, M. (1990): "Precautionary saving in the small and in the large," Econometrica, 58, 53-73. 
Kolm, S.-C. (1969): "The Optimal Production of Social Justice," in Public Economics, ed. by J. Margolis, and H. Guitton, London. Macmillan.

(1976): "Unequal Inequalities II," Journal of Economic Theory, 13, 82-111.

Mehran, F. (1976): "Linear measures of income inequality," Econometrica, 44, 805-809.

QuigGin, J. (1982): "A theory of anticipated utility," Journal of Economic Behavior and Organization, 3, 323-343.

Sen, A. (1973): On Economic Inequality. Clarendon Press, Oxford.

Shorrocks, A., And J. Foster (1987): “Transfer Sensitive Inequality Measures," Review of Economic Studies, 54, 485-497.

Weymark, J. (1981): "Generalized Gini Inequality Indices," Mathematical Social Sciences, 1, 409-430.

YAARI, M. (1987): "The dual theory of choice under risk," Econometrica, 55(1), 95-115.

_ (1988): "A controversal proposal concerning inequality measurement," Journal of Economic Theory, 44, 381-97.

ZoLI, C. (1999): "Intersecting generalized Lorenz curves and the Gini index," Social Choice and Welfare, 16, 183-196. 